within a few days of each other, without suggesting the idea of plague unless that disease is in one's mind. A woman in the pregnant state may abort and die (the usual sequence in plague), a child may be suddenly convulsed, glandular fever may occur amongst children, or a patient may develop abdominal symptoms of a typhoid character, without the fact of plague being the cause occurring to anyone. But when plague exists in a locality the diagnosis of a typical case is easy. The pneumonic type of the disease is, however, difficult of diagnosis, especially if the thought of plague is not present in one's mind. Assistance in suspecting plague in such cases is afforded by the rapid onset of the lung lesion without any previous bronchial symptoms, by the sudden attack of prostration, fever, and general symptoms, and by inspection of the sputum, which will be found watery and profuse, and tinged by or mixed with blood. It is only by microscopic examination of the sputum, however, that the presence of the disease can be actually diagnosed. In fact, the diagnosis of no case of plague is complete unless the bacillus pestis is proved to exist. In the pneumonic form the diagnosis may readily be made by microscopic examination of cover-glass preparations prepared from the sputum. In the bubonic form all that one has to do to obtain a specimen is to make a small incision into the gland, the skin having been previously rendered aseptic. Anæsthesia may be obtained by freezing, but no chemical antiseptics should be employed in the operation, except for the preliminary cleansing of the skin. A small piece of the gland tissue should be placed at once into a sterilised test-tube. Broth tubes may also be inoculated, and cover-glass preparations made if these are at hand, and the whole should at once be sent to a laboratory for examination, the case in the meantime being isolated. If incision of the glands is not thought desirable, blood films may be obtained by hypodermic puncture of the buboes. The needle used should afterwards be at once destroyed by fire. To put the whole thing in a nutshell, what all medical men have to look out for jusi now is fever with glandular swellings. In the last report drawn up by the late Sir Richard Thorne, he said: "Fever with glandular swellings prevailed in Bombay before it was recognised that plague had reached that city; and it is impossible to read the medical history of this disease in almost every part of the world, without being impressed with the frequency with which recognised plague has been preceded by ailments of such slight severity, involving some bubonic enlargement of glands, and some rise in body temperature, as to mask the real nature of the malady." Fever, then, of whatever type, complicated with glandular swelling of whatever intensity, is what medical practitioners all over the country have to look out for: in addition to which all ill-defined febrile attacks should be carefully investigated.

\section{SUB-DIAPHRAGMATIC ABSCESS.}

The space below the diaphragm is one which is not easy to get at, either for diagnosis or for treatment, and when an abscess forms there it becomes a question of serious urgency to determine how to give relief.

A good review of the whole subject of sub-diaphragmatic abscess was given at the Ipswich meeting of the British Medical Association. After giving a long list of the causes by which abscess in this region might be produced, Mr. Rickman Godlee pointed out the important fact that subphrenic abscess is not an isolated disease occurring on its own account, but is almost of necessity a complication of something else. It is therefore by no means easy to isolate the symptoms due to it, and say that such and such are the symptoms by which its presence can be diagnosed.

Being an accident or by-product of some other disease, the symptoms of the latter will probably be by far the most prominent ones, and may by their aggressiveness lead to the abscess being overlooked. Say, for example, that the disease starts as an appendicitis, and that the lump in the groin subsides as the abscess tracks up outside the ascending colon, and spreads out under the diaphragm, how easily it may be overlooked or mistaken for a secondary pleural effusion.

To show how impossible it is to lay down any definite series of symptoms as indicating without doubt the presence of sub-diaphragmatic abscess, one has but to point to the variety in shape and size which such an abscess may assume, the very different positions it may occupy, and the very numerous diseases by which it may be caused. Such abscesses may be divided primarily into extraperitoneal, as when they start from caries of the spine or ribs, or spread up from the kidneys, and intraperitoneal, which is by far the commoner form, when they start from the rupture of an abscess in one of the viscera below the diaphragm. "Think only," said Mr. Godlee, "of such a case as that of a very chronic ulcer of the stomach which has caused extensive adhesions between the viscus and the diaphragm; an abscess may form amongst these adhesions and burrow about by complicated and narrow tracks until it points perhaps through an intercostal space. Then contrast with it one in which a sudden perforation of a stomach ulcer has occurred, but in which the suppuration is to some extent localised and contains air. It is difficult to trace a family resemblance between these two conditions."

Subphrenic abscess may be defined as any collection of pus, or of pus and gas, the whole or part of which intervenes between the diaphragm and the structures normally in contact with it. The great point in regard to diagnosis is to separate the disease from pleurisy, from which, however, it ought to be distinguishable by the following points :-

(a) The movements of the chest are not impaired on the affected side.

(b) The upper limit of dulness is not so sharply defined.

(c) Breath sounds may be heard below the level of the dulness, and if a deep inspiration be made the line at which the breath sounds and vocal resonance are heard, and at which vocal fremitus is felt, is distinctly lowered.

(d) When there is much emaciation the lower border of the lung may sometimes be distinctly seen moving up and down during respiration, showing clearly that the cause of the dulness is below and not above the diaphragm.

Instead of dulness there may be a great extent of tympanitic resonance, and over the resonant area the "bell sound" may be elicited in a most perfect manner from the presence of gas as well as pus. From the similarity of the signs to those of pneumothorax this condition has been called "subdiaphragmatic pneumothorax," and it is surprising how high this tympanitic area (or the dull area for the matter of that) may extend, rising up as high as the second rib and reaching down into the iliac fossa.

Among the important points raised in the discussion may be mentioned one made by Mr. Bidwell, to the effect that a peculiarly offensive character in the pus withdrawn from an empyæma, and the fact that an empyæma does 
not improve after resection of the rib and irrigation, should make one suspect the presence of subdiaphragmatic abscess ; and one made by Mr. Bruce Clarke, who pointed out how great an aid to diagnosis was invariably given in cases of doubt by introducing the hand into the peritoneal cavity and investigating fully the abnormal condition.

THE CHOICE OF OPERATION FOR THE REMOVAL OF LARGE STONES FROM THE BLADDER.

IN a recent discussion at Ipswich as to the best treatment of large vesical calculi, Mr. Cadge, of Norwich, made some interesting remarks in regard to the choice of operation in such cases, and in so doing he drew a broad distinction between the proceedings which might properly be undertaken by specialists who had had the advantage of considerable practical experience in operating in such cases, and general hospital surgeons who only treated these cases occasionally, saying that while the expert in stone practice might be trusted to do what he thought best, what might seem best in his experienced hands might not be best in the hands of the general surgeon. He said that at the present day the question as to the treatment of large stones seemed to lie between suprapubic cystotomy and perineal lithotrity, or rather litholapaxy, and that while to the specialist the latter might be the best, to the ordinary surgeon the former was the preferable operation. It is, he says, easier of execution than any form of perineal operation; the parts dealt with are under the eye ; there are no vessels likely to be met with which will give trouble; the bladder wall to be cut can be incised to any extent, and stitched up after the stone's removal if necessary; moreover, an enlarged prostate which so often impedes perineal operations causes no difficulty, or very slight, in the suprapubic method; while, should a stone be fixed in the end of the ureter, or in some form of sac, it can be dealt with far more easily than by any other opening.

Several surgeons, however, who had devoted considerable attention to the subject, differed very strongly from the recommendations made by $\mathrm{Mr}$. Cadge, and $\mathrm{Mr}$. Freyer was particularly scornful. But we may be allowed to doubt whether the statistical argument which was especially relied upon by some can properly decide the question, and it must also be pointed out that the problem discussed by Mr. Cadge was not Which is the best operation? but, Which is the best for a man to perform who, from the circumstances, can only bave a comparatively small operative experience in such cases? The really good point made against the suprapubic operation was that mentioned by Mr. Reginald Harrison, who showed that where there is a tendency to the formation of stone, calculous material may be deposited again and again upon the scar left by the high operation. This is a real objection, and one of great, interest, and if it should be found to be operative in many cases it would form a great bar to the utility of the suprapubic cystotomy.

We cannot hide from ourselves that the whole question is one of considerable interest quite outside the mere surgical problem involved. It seems now to be definitely maintained by certain specialists that success in operating for stone depends on the use of special instruments - which of course are open to anyone-and on the possession of special experience which can only be obtained by few. This raises an ethical problem which it would require some hardihood to discuss in any medical society-where the majority of surgeons present could probably count upon their fingers the stones on which they had operatednamely, Should the general hospital surgeon undertake such cases any more than he would undertake operations, say, for cataract? The mere hinting of such a question may seem to some to savour of specialism gone mad. But it is a thing which is in the air about many operations, and will sooner or later have to be seriously discussed.

\section{Progress in Psychiatry.}

\section{(Continued from page 386 , vol. xxviii.)}

Toxic Action of Morphia.-Morphia causes insanity more rarely than the other toxic substances which are indulged in by mankind for purposes of pleasure and for experiencing temporary feelings of well-being (euphoria). Its prolonged abuse gives rise to psychical disorders (disorders of personality) that confine themselves to the "domain of semi-alienation" (Regis) - the borderland between sanity and insanity. Opium seems to be the intoxicant which, next to alcohol, has been indulged in most largely by the human race. Its abuse as a narcotic is traceable to early historic times. Murrell has shown that the inhabitants of the fens of Lincolnshire had long employed opium as a prophylactic against malaria. The same practice (according to Talbot ${ }^{58}$ ) prevailed in certain malarial regions of New Jersey and Pennsylvania, where the use of strong infusion of poppy was common. A tendency to opium-tolerance appears to have been observed in the children of parents, especially mothers, addicted to the opium habit. The fotus in utero receives the toxic agent through the placenta, just as it is known to receive alcohol from an inebriated mother. In consequence, the child in the first months of infancy must be nourished on the milk of an opium-using woman, or given opium in some other way, lest it perish. ${ }^{9}$ To this fact Calkins ${ }^{c 0}$. was the first to call attention, and his results were confirmed by Erlenmeyer of Berlin, Mattison of
Brooklyn, Kiernan ${ }^{c 1}$ of Chicago, and others. The lastmentioned author, after quoting numerous instances of the congenital opium-habit where opium was needed to preserve the infant during the first months of life, points out that pigeons whose ancestors were fed on poppies became tolerant of opium. Murrell found that the same was relatively true of persons descended from ancestors (in Bedfordshire) who used infusions of poppy as a prophylactic against malaria. It appears, moreover, that the children of parents addicted to morphia were often still-born or were the victims of some form of cerebral and mental defect, with a marked tendency to infantile convulsions (Amabile), or to congenital idiocy and imbecility and other forms of cerebral degeneration. Levinstein has shown by experiments on pregnant bitches and rabbits that the use of opium during pregnancy produced either abortion or still-births, or rapidly-dying offspring. These facts regarding the far-reaching deleterious effects of opium, both on the parent and the offspring, suffice to show its resemblance to alcohol as a toxic agent (Hospital, December 30th, 1899, pp. 211-12).

The opium-eater and the morphia habitue indulge in these drugs for the same reason, viz.: to experience pleasure and euphoria in the first instance. Agreeable illusions and hallucinations of an erotic nature are attributed to opium, but these do nersist in the later 